\title{
FROM ISOTONIC BANACH FUNCTIONALS TO COHERENT RISK MEASURES
}

Abstract. Coherent risk measures $[\mathrm{ADEH}]$, introduced to study both market and nonmarket risks, have four characteristic properties that lead to the term "coherent" present in their name. Coherent risk measures regarded as functionals on the space $L^{\infty}(\Omega, \mathcal{F}, \mathbb{P})$ have been extensively studied $[\mathrm{De}]$ with respect to these four properties. In this paper we introduce CRM functionals, defined as isotonic Banach functionals [Al], and use them to characterize coherent risk measures on the space $L^{\infty}(\Omega, \mathcal{F}, \mathbb{P})$ as order opposites of CRM functionals. The characterization involves only three axioms and leaves room for a larger class of functionals that can be related to a larger class of possible risks. We show that every CRM functional, when restricted to constant functions, is represented by a convex real function on $\mathbb{R}$ which is linear for nonnegative and nonpositive arguments separately. Next, we show that those CRM functionals which are extensions of the map $\mathbb{R} \ni t \mapsto \beta t \in \mathbb{R}$, with $\beta>0$, are represented as maxima over a set of positive linear extensions.

1. Introduction. Let $(\Omega, \mathcal{F}, \mathbb{P})$ denote a fixed probability space, and $L^{\infty}(\Omega, \mathcal{F}, \mathbb{P})$ be the Banach space of all (equivalence classes of) real-valued bounded measurable functions with the essential supremum norm, denoted by $\|\cdot\|_{\infty}$. Its (topological) dual is the Banach space $\mathbf{b a}(\Omega, \mathcal{F}, \mathbb{P})$ of all realvalued finitely additive measures $\mu$ on the measurable space $(\Omega, \mathcal{F})$ with the property that $\mathbb{P}(A)=0$ implies $\mu(A)=0$. The norm of any $\mu$ in this space is given by its total variation $[\mathrm{YH}],[\mathrm{Al}],[\mathrm{Gr}],[\mathrm{Bo}]$. For brevity, we will refer to these spaces as $L^{\infty}(\mathbb{P})$ or just $L^{\infty}$, and respectively ba $(\mathbb{P})$ or just ba. A coherent risk measure $[\mathrm{De}]$ is a map $\varrho: L^{\infty}(\Omega, \mathcal{F}, \mathbb{P}) \rightarrow \mathbb{R}$ that satisfies the following four conditions:

2000 Mathematics Subject Classification: 46A22, 91B30.

Key words and phrases: coherent risk measure, isotonic functional. 
(1.1) If $X \geq 0$ then $\varrho(X) \leq 0$ for all $X \in L^{\infty}(\mathbb{P})$.

(1.2) $\quad \varrho(X+Y) \leq \varrho(X)+\varrho(Y)$ for all $X, Y \in L^{\infty}(\mathbb{P})$.

(1.3) $\varrho(\lambda X)=\lambda \varrho(X)$ for all $\lambda \geq 0$ and all $X \in L^{\infty}(\mathbb{P})$.

(1.4) $\varrho(X+\alpha)=\varrho(X)-\alpha$ for all $X \in L^{\infty}(\mathbb{P})$ and all scalars $\alpha \in \mathbb{R}$.

We are going to characterize coherent risk measures in terms of isotonic Banach functionals and extensions of positive linear functionals, and in order to do so we shall require the following definitions.

Definition 1.1. Given a map $F: L^{\infty}(\mathbb{P}) \rightarrow \mathbb{R}$, we say that a real-valued function $\varphi: \mathbb{R} \rightarrow \mathbb{R}$ is a scalar base of $F$ if

$$
\varphi(\alpha)=F(\alpha 1) \quad \text { for all } \alpha \in \mathbb{R},
$$

where 1 denotes the constant function equal to 1 for every $\omega \in \Omega$.

Definition 1.2. Given two maps $\kappa, \lambda: L^{\infty}(\mathbb{P}) \rightarrow \mathbb{R}$, we say that $\lambda$ is the order opposite of $\kappa$ if

$$
\lambda(X)=\kappa(-X) \quad \text { for all } X \in L^{\infty}(\mathbb{P}) .
$$

COROLlaRY 1.1. The map $\lambda$ is the order opposite of $\kappa$ iff $\kappa$ is the order opposite of $\lambda$.

The identity (SBF) means that the function $\varphi$ is the restriction of the functional $F$ to the subspace of constant functions in $L^{\infty}(\mathbb{P})$. It will turn out that coherent risk measures are determined to a large degree by scalar bases of their order opposites.

Proposition 1.1. Let $\varrho: L^{\infty}(\mathbb{P}) \rightarrow \mathbb{R}$ be a coherent risk measure and $L_{\varrho}$ be its order opposite. Then the functional $L_{\varrho}$ satisfies the following four conditions:

$\left(1.2^{\prime}\right) \quad L_{\varrho}(X+Y) \leq L_{\varrho}(X)+L_{\varrho}(Y)$ for all $X, Y \in L^{\infty}(\mathbb{P})$ (subadditivity). $\left(1.3^{\prime}\right) \quad L_{\varrho}(\lambda X)=\lambda L_{\varrho}(X)$ for all $\lambda \geq 0$ and all $X \in L^{\infty}(\mathbb{P})$ (positive homogeneity).

$\left(1.4^{\prime}\right) \quad L_{\varrho}(X+\alpha)=L_{\varrho}(X)+\alpha$ for all $X \in L^{\infty}(\mathbb{P})$ and all scalars $\alpha \in \mathbb{R}$.

Proof. Obvious.

Proposition 1.2. Let $L: L^{\infty}(\mathbb{P}) \rightarrow \mathbb{R}$ satisfy conditions $\left(1.1^{\prime}\right)-\left(1.4^{\prime}\right)$ and $\varrho_{L}$ be its order opposite. Then the functional $\varrho_{L}$ is a coherent risk measure.

Proof. Obvious.

Proposition 1.3. Let $L: L^{\infty}(\mathbb{P}) \rightarrow \mathbb{R}$ be a functional that satisfies conditions $\left(1.1^{\prime}\right)-\left(1.4^{\prime}\right)$. Then

$$
L(0)=0 .
$$

$$
L(\alpha)=\alpha \text { for any constant function } \alpha \text {. }
$$


(1.8) $\quad L(X-L(X))=0$.

(1.9) $0 \leq X$ implies $0 \leq L(X)$.

(1.10) $\quad X \leq Y$ implies $L(X) \leq L(Y)$.

(1.11) $\quad \alpha \leq X \leq \beta$ implies $\alpha \leq L(X) \leq \beta$.

(1.12) $|L(X-Y)| \leq\|X-Y\|_{\infty}$.

(1.13) $|L(X)-L(Y)| \leq\|X-Y\|_{\infty}$.

Proof. First, (1.6) follows from $\left(1.3^{\prime}\right)$ with $\lambda$ set to $0 ;(1.7)$ follows from $\left(1.4^{\prime}\right)$ and $(1.6) ;(1.8)$ follows from $(1.7)$ and $\left(1.4^{\prime}\right) ;(1.9)$ follows from the fact that $0 \leq X$ implies $-X \leq 0$ and that $0=L(X-X) \leq L(X)+L(-X) \leq$ $L(X)$.

Regarding (1.10), clearly $X \leq Y$ implies $X-Y \leq 0$, and so

$$
L(X)=L((X-Y)+Y) \leq L(X-Y)+L(Y) \leq L(Y) .
$$

The property (1.11) follows directly from (1.10); (1.12) follows from (1.11) and the fact that

$$
-\|X\|_{\infty} \leq X \leq\|X\|_{\infty} \quad \text { and } \quad-\|X\|_{\infty} \leq-X \leq\|X\|_{\infty}, \mathbb{P} \text {-a.e. }
$$

Similarly, by (1.14), we have

$$
\begin{aligned}
& L(X)-L(Y) \leq L(X-Y) \leq\|X-Y\|_{\infty}, \\
& L(Y)-L(X) \leq L(Y-X) \leq\|X-Y\|_{\infty} .
\end{aligned}
$$

Therefore, (1.13) also holds.

The properties (1.6)-(1.13) have their counterparts for coherent risk measures. It follows from the above that we can characterize coherent risk measures in terms of functionals that satisfy conditions $\left(1.1^{\prime}\right)-\left(1.4^{\prime}\right)$ and of their order opposites, and vice versa. Both types of functionals are subadditive and positively homogeneous, that is, both are Banach functionals [Al, p. 92]. Both types are convex, norm continuous, and linear on constant functions. The main difference is that coherent risk measures map the cone of positive elements into its opposite, while their order inverses preserve the order, that is, they are isotonic [Al, p. 503]. Since the positive (linear) functionals have already proven to be a valid tool for exploring duality problems involving partially ordered spaces, we choose their nonlinear counterpart as a tool to explore the general properties of coherent risk measures.

2. Isotonic Banach functionals. We can characterize the functionals that satisfy the conditions $\left(1.1^{\prime}\right)-\left(1.4^{\prime}\right)$ with a somewhat simpler set of conditions.

Theorem 2.1. A map $L: L^{\infty}(\Omega, \mathcal{F}, \mathbb{P}) \rightarrow \mathbb{R}$ satisfies $\left(1.1^{\prime}\right)-\left(1.4^{\prime}\right)$ iff it satisfies the following three conditions:

(CRM1) If $X \leq Y$ then $L(X) \leq L(Y)$, for all $X, Y \in L^{\infty}(\mathbb{P})$. 
(CRM2) $\quad L(\alpha X+\beta Y) \leq \alpha L(X)+\beta L(Y)$ for all $X, Y \in L^{\infty}(\mathbb{P})$ and $\alpha, \beta \geq 0$.

(CRM3) $L(-1)=-1=-L(1)$.

Proof. First, we show that (CRM2) alone implies $L(0)=0$. Setting $\alpha=$ $\beta=0$ in $(\mathrm{CRM} 2)$, we get $L(0) \leq 0$. On the other hand, setting $\alpha=\beta=1$ and $X=Y=0$ in (CRM2), we get $L(0)=L(0+0) \leq L(0)+L(0)$, so that $0 \leq L(0)$. Therefore,

$$
L(0)=0 \quad \text { if } L \text { satisfies (CRM2). }
$$

Now, assume that $L$ satisfies $\left(1.1^{\prime}\right)-\left(1.4^{\prime}\right)$. Note that $X \leq Y$ implies $X-Y \leq 0$, so that

$$
L(X)=L(X-Y+Y) \leq L(X-Y)+L(Y) \leq L(Y) .
$$

Thus (CMR1) holds. (CMR2) follows directly from $\left(1.2^{\prime}\right)$ and $\left(1.3^{\prime}\right)$. Setting $X=0$ in $\left(1.4^{\prime}\right)$ and using (2.1) we get (CRM3).

Conversely, assume that $L$ satisfies (CRM1)-(CRM3). Then condition $\left(1.1^{\prime}\right)$ follows directly from (CRM1). Setting $\alpha=\beta=1$ in (CRM2) we get $\left(1.2^{\prime}\right)$.

To get $\left(1.3^{\prime}\right)$ we consider two cases. First, let $\lambda=0$. Then, by (2.1), we obtain

$$
L(0 \cdot X)=L(0)=0=0 \cdot L(X),
$$

that is, $\left(1.3^{\prime}\right)$ holds for $\lambda=0$. Second, let $\lambda>0$. Then, setting $\alpha=\lambda$ and $\beta=0$ in (CRM2), we get

$$
L(\lambda X) \leq \lambda L(X)
$$

On the other hand,

$$
L(X)=L[(1 / \lambda) \lambda X] \leq(1 / \lambda) L(\lambda X) \leq(1 / \lambda) \lambda L(X)=L(X)
$$

so that $L(X)=(1 / \lambda) L(\lambda X)$. Therefore $\left(1.3^{\prime}\right)$ holds for all $\lambda \geq 0$.

Now, it remains to show that $\left(1.4^{\prime}\right)$ holds. Clearly, by (CRM3) and $\left(1.3^{\prime}\right)$, for arbitrary $\alpha \in \mathbb{R}$ we have $L(\alpha)=\alpha$. Next, for every $X \in L^{\infty}(\mathbb{P})$ and constant function $\alpha$,

$$
L(X+\alpha) \leq L(X)+L(\alpha)=L(X)+\alpha .
$$

On the other hand,

$$
L(X)+\alpha=L[(X+\alpha)-\alpha]+\alpha \leq L(X+\alpha)+L(-\alpha)+\alpha=L(X+\alpha) .
$$

Therefore, $L(X+\alpha)=L(X)+\alpha$, that is, $\left(1.4^{\prime}\right)$ holds. This completes the proof.

Corollary 2.1. A map $L: L^{\infty}(\mathbb{P}) \rightarrow \mathbb{R}$ satisfies (CRM2) iff it is a Banach functional. 
The condition (CRM1) means that the functional $L$ is isotonic [Al, p. 503]. The condition (CRM2) means that it is positively sublinear, or a Banach functional, and (CRM3) assigns specific values to the constant functions 1 and -1 , respectively.

Following the above characterization, we adopt the following definition.

Definition 2.1. A map $L: L^{\infty}(\mathbb{P}) \rightarrow \mathbb{R}$ is a CRM functional if it satisfies the following conditions:

If $X \leq Y$ then $L(X) \leq L(Y)$, for all $X, Y \in L^{\infty}(\mathbb{P})$ (isotonicity). $L(X+Y) \leq L(X)+L(Y)$ for all $X, Y \in L^{\infty}(\mathbb{P})$ (subadditivity). $L(\lambda X)=\lambda L(X)$ for all $\lambda \geq 0$ and all $X \in L^{\infty}(\mathbb{P})$ (positive homogeneity).

Corollary 2.2. A map $L: L^{\infty}(\mathbb{P}) \rightarrow \mathbb{R}$ is a CRM functional iff it satisfies the conditions (CRM1) and (CRM2).

Proposition 2.1. Let $L: L^{\infty}(\mathbb{P}) \rightarrow \mathbb{R}$ be a CRM functional and $\varphi$ its scalar base. Then there are two nonnegative constants $\beta^{+}$and $\beta^{-}$such that $\beta^{+} \geq \beta^{-}$and

$$
\varphi(t)= \begin{cases}\beta^{+} t & \text { if } t \geq 0, \\ \beta^{-} t & \text { if } t \leq 0 .\end{cases}
$$

Proof. By (BA2), $\varphi(t)=t \varphi(1)$ for $t \geq 0$. Set $\beta^{+}=\varphi(1)=L(1) \geq 0$, as $1 \geq 0$. Similarly, $\varphi(t)=|t| \varphi(-1)$ for $t \leq 0$. Moreover, since $L(-1) \leq 0$, choosing $\beta^{-}=-\varphi(-1)=-L(-1)$, we get $\beta^{-} \geq 0$, and (2.2) holds.

Choosing $X=-1$ and $Y=1$, and $\alpha=\beta=1$, by (BA1) we get

$$
\varphi(0)=0=L(-1+1) \leq L(-1)+L(1)=\varphi(-1)+\varphi(1)=-\beta^{-}+\beta^{+} .
$$

This ends the proof.

CoROllary 2.3. The scalar base of an arbitrary CRM functional $L$ is a convex function $\varphi: \mathbb{R} \rightarrow \mathbb{R}$ such that (i) $\varphi(0)=0$; (ii) $\varphi$ is nondecreasing; (iii) $\varphi$ is linear on the infinite intervals $(-\infty, 0]$ and $[0, \infty)$ (separately).

The behaviour of CRM functionals on the subspace of constant functions determines to a large degree the functionals themselves, as illustrated by the following.

Proposition 2.2. Let $L: L^{\infty}(\mathbb{P}) \rightarrow \mathbb{R}$ be a CRM functional. Assume that $L\left(\alpha_{0}\right)=0$ for a nonzero scalar $\alpha_{0}$. Then

$$
\begin{array}{lll}
L(X)=0 & \text { for all } X \in L^{\infty}(\mathbb{P}) & \text { if } \alpha_{0}>0, \\
L(X)=0 & \text { for all } X \in L^{\infty}(\mathbb{P}) \text { such that } X \leq 0 & \text { if } \alpha_{0}<0 .
\end{array}
$$

Proof. Let $\varphi$ denote the scalar base of $L$, with the corresponding constants $\beta^{+} \geq \beta^{-} \geq 0$. If $\alpha_{0}>0$, then $\beta^{+} \alpha_{0}=0$, so that $\beta^{+}=0$, and consequently $L(t 1)=\varphi(t)=0$ for all scalars $t$. Therefore, for every $X \in L^{\infty}(\mathbb{P})$, 
we have $-\|X\|_{\infty} \leq X \leq\|X\|_{\infty}$, and so

$$
0=L\left(-\|X\|_{\infty}\right) \leq L(X) \leq L\left(\|X\|_{\infty}\right)=0
$$

which proves (2.3).

If $\alpha_{0}<0$, then $\beta^{-} \alpha_{0}=0$, so that $\beta^{-}=0$, and consequently $L(t 1)=$ $\varphi(t)=0$ for all nonpositive scalars $t$. Therefore, for every nonpositive $X \in$ $L^{\infty}(\mathbb{P})$, we have $-\|X\|_{\infty} \leq X \leq 0$, and so $0=L\left(-\|X\|_{\infty}\right) \leq L(X) \leq 0$, which proves $(2.4)$.

Of course, every positive linear functional $l: L^{\infty}(\mathbb{P}) \rightarrow \mathbb{R}$ is a CRM functional. A less trivial example is given by the following.

EXAmPLE 2.1. Let $l_{1}, l_{2}: L^{\infty}(\mathbb{P}) \rightarrow \mathbb{R}$ be two positive linear functionals. Define a functional $L: L^{\infty}(\mathbb{P}) \rightarrow \mathbb{R}$ as follows:

$$
L(X)=\max \left(l_{1}(X), l_{2}(X)\right) \quad \text { for } X \in L^{\infty}(\mathbb{P}) .
$$

The functional (2.5) is isotonic since $X \leq Y$ implies that

$$
L(X)=\max \left(l_{1}(X), l_{2}(X)\right) \leq \max \left(l_{1}(Y), l_{2}(Y)\right)=L(Y) .
$$

It is also subadditive since for any $X, Y \in L^{\infty}(\mathbb{P})$, we have

$$
\begin{aligned}
L(X+Y) & =\max \left(l_{1}(X+Y), l_{2}(X+Y)\right) \\
& =\max \left(l_{1}(X)+l_{1}(Y), l_{2}(X)+l_{2}(Y)\right) \\
& \leq \max \left(l_{1}(X), l_{2}(X)\right)+\max \left(l_{1}(Y)+l_{2}(Y)\right) \\
& =L(X+Y) .
\end{aligned}
$$

Its positive homogeneity is obvious, so $L$ is indeed a CRM functional.

Let $\operatorname{CRM}(\mathbb{P})$ denotes the set of all CRM functionals on $L^{\infty}(\mathbb{P})$. It can be regarded as a subset of the ordered linear space $\operatorname{Map}\left(L^{\infty}(\mathbb{P}), \mathbb{R}\right)$ of all realvalued functions $L^{\infty}(\mathbb{P}) \rightarrow \mathbb{R}$, with the standard operations and partial order defined by the cone of positive functions (that is, all nonnegative mappings from $L^{\infty}(\mathbb{P})$ into $\left.\mathbb{R}\right)$. Then it is clear that the set $\operatorname{CRM}(\mathbb{P})$ is closed with respect to (i) addition; (ii) multiplication by nonnegative constants; and (iii) the operation of taking a maximum. That is, we have the following.

Proposition 2.3. (i) $\operatorname{CRM}(\mathbb{P})+\operatorname{CRM}(\mathbb{P}) \subset \operatorname{CRM}(\mathbb{P})$.

(ii) $\lambda \mathrm{CRM}(\mathbb{P}) \subset \mathrm{CRM}(\mathbb{P})$ for every $\lambda \geq 0$.

(iii) $F, G \in \operatorname{CRM}(\mathbb{P}) \Rightarrow \max (F, G) \in \operatorname{CRM}(\mathbb{P})$.

The last proposition says that the set of all CRM functionals on $L^{\infty}(\mathbb{P})$ is a convex cone which is closed with respect to the maximum operation. Actually it is closed with respect to taking the supremum of any collection of CRM functionals provided the supremum is finite for all elements of $L^{\infty}(\mathbb{P})$. 
Proposition 2.4. Let $\left\{f_{\alpha}\right\}_{\alpha \in A}$ be a subset of $\operatorname{CRM}(\mathbb{P})$ bounded from above by a functional $g: L^{\infty}(\mathbb{P}) \rightarrow \mathbb{R}$. Then the functional defined by

$$
f(X)=\sup \left\{f_{\alpha}(X) \mid \alpha \in A\right\} \quad \text { for } X \in L^{\infty}(\mathbb{P})
$$

is also a CRM functional.

Proof. This follows directly from the definition and from the properties of the supremum.

By the Hahn-Banach like arguments, the converse is also true for CRM functionals whose scalar base is a (positive) linear functional on the set of real numbers.

First, we will prove the following fact.

TheOREM 2.2. Let $F: L^{\infty}(\mathbb{P}) \rightarrow \mathbb{R}$ be a CRM functional with a linear scalar base. Then for every $X_{0} \in L^{\infty}(\mathbb{P})$ there exists a linear CRM functional $L: L^{\infty}(\mathbb{P}) \rightarrow \mathbb{R}$ such that

$$
\begin{aligned}
L\left(X_{0}\right) & =F\left(X_{0}\right), \\
L(X) & \leq F(X) \quad \text { for all } X \in L^{\infty}(\mathbb{P}) .
\end{aligned}
$$

Proof. If $X_{0}=0$, or $F=0$, the conclusion trivially holds, so assume that $X_{0} \neq 0$, and that $F$ is nontrivial. Assume also, for simplicity, that $F(1)=-F(-1)=1$.

Next, consider the subspace $\mathcal{Y}_{0}$ of $L^{\infty}(\mathbb{P})$ spanned by the elements $X_{0}$ and 1 . Let $l_{0}: \mathcal{Y}_{0} \rightarrow \mathbb{R}$ be the linear functional defined as follows:

$$
l_{0}\left(\alpha X_{0}+\beta 1\right)=\alpha F\left(X_{0}\right)+\beta \quad \text { for } \alpha, \beta \in \mathbb{R} .
$$

Then, of course, $l_{0}(1)=1$ and $l_{0}\left(X_{0}\right)=F\left(X_{0}\right)$.

Now we show that the functional (2.8) is positive. So, let $\alpha, \beta \in \mathbb{R}$ be such that $\alpha X_{0}+\beta 1 \geq 0$. Then for $\alpha \geq 0$ we have

$$
l_{0}\left(\alpha X_{0}+\beta 1\right)=\alpha F\left(X_{0}\right)+\beta=F\left(\alpha X_{0}\right)+\beta=F\left(\alpha X_{0}+\beta 1\right) \geq 0 .
$$

Next, for $\alpha<0$ we have

$$
X_{0}+\frac{\beta}{\alpha} 1 \leq 0
$$

so that

$$
F\left(X_{0}\right)+\frac{\beta}{\alpha} \leq 0
$$

and thus $0 \leq \alpha F\left(X_{0}\right)+\beta=l_{0}\left(\alpha X_{0}+\beta 1\right)$.

Now, we will show that $l_{0}$ is bounded by $F$ on $\mathcal{Y}_{0}$. Let $\alpha X_{0}+\beta 1 \in \mathcal{Y}_{0}$. For $\alpha \geq 0$ we have, by (2.9),

$$
l_{0}\left(\alpha X_{0}+\beta 1\right)=F\left(\alpha X_{0}+\beta 1\right) \leq F\left(\alpha X_{0}+\beta 1\right) .
$$


For $\alpha<0$ we have

$$
\begin{aligned}
l_{0}\left(\alpha X_{0}+\beta 1\right) & =\alpha F\left(X_{0}\right)+\beta=\frac{\alpha}{|\alpha|} F\left(|\alpha| X_{0}\right)+\beta \\
& =-F\left(|\alpha| X_{0}\right)+\beta \leq F\left(-|\alpha| X_{0}\right)+\beta=F\left(\alpha X_{0}+\beta 1\right) .
\end{aligned}
$$

Therefore, by the Hahn-Banach theorem, there exists a linear extension of $l_{0}$, say $L$, to all of $L^{\infty}(\mathbb{P})$, such that

$$
L(X) \leq F(X) \quad \text { for all } X \in L^{\infty}(\mathbb{P}) .
$$

Thus, it remains to show that the functional $L$ is positive. So, let $X \in$ $L^{\infty}(\mathbb{P})$ and let $0 \leq X$. Then $-X \leq 0$ and

$$
L(-X) \leq F(-X) \leq 0 .
$$

This implies that

$$
0 \leq-F(-X) \leq L(X) .
$$

Therefore, the functional $L$ is positive, and thus it is a CRM functional.

Looking at (2.10) and (2.11) we can see that in proving the positivity of $L$ we have only used the fact that $L$ is bounded by the CRM functional $F$. This leads to the following remarkable and unexpected fact.

COROLlary 2.3. Every linear functional bounded by a CRM functional is a CRM functional.

Now, we can prove the announced theorem on maximization of a CRM functional with a linear scalar base by positive linear functionals it bounds from above.

Theorem 2.3. If $F: L^{\infty}(\mathbb{P}) \rightarrow \mathbb{R}$ is a $C R M$ functional such that $F(1)=$ $-F(-1)=\kappa>0$, then there is a convex set $\mathcal{L}$ of positive linear functionals such that

$$
\begin{gathered}
\mathcal{L} \leq F, \\
\mu \in \mathcal{L} \Rightarrow \mu(1)=\kappa, \\
F(X)=\max \{\mu(X) \mid \mu \in \mathcal{L}\} .
\end{gathered}
$$

Proof. We can assume that $\kappa=1$. Otherwise we would consider the functional $F^{\prime}$ given by $F^{\prime}(X)=F(X / \kappa)$. Define $\mathcal{L}$ to be the set of all positive linear extensions of the scalar base of the functional $F$ to all of $L^{\infty}(\mathbb{P})$, and bounded from above by $F$. The set $\mathcal{L}$ is nonempty by Theorem 2.2. By definition, it satisfies (2.12).

It is convex, since for any $\mu, \nu \in \mathcal{L}$, and for any $a, b \geq 0$ such that $a+b=1$, we get

$$
(a \mu+b \nu)(1)=a \mu(1)+b \nu(1)=a \kappa+b \kappa=(a+b) \kappa=\kappa .
$$


Also, it follows from Theorem 1.2 that

$$
F(X) \leq \sup \{\mu(X) \mid \mu \in \mathcal{L}\},
$$

since for every $X \in L^{\infty}(\mathbb{P})$, there is $\mu_{0} \in \mathcal{L}$ such that $F(X)=\mu_{0}(X)$.

On the other hand, for every $X \in L^{\infty}(\mathbb{P})$ and $\mu \in \mathcal{L}, \mu_{0}(X) \leq F(X)$, so

$$
F(X) \geq \sup \{\mu(X) \mid \mu \in \mathcal{L}\}
$$

and thus (2.14) holds, because by (2.6) we can replace sup by max. This ends the proof.

Corollary 2.4. The set $\mathcal{L}$ in Theorem 2.3 is $\sigma\left(\mathbf{b a}, L^{\infty}\right)$-closed.

Proof. By definition,

$$
\mathcal{L}=\{\mu \in \mathbf{b a}(\mathbb{P}) \mid \mu(1)=\kappa, 0 \leq \mu\} \quad \text { (where } \kappa \geq 0 \text { is fixed) } .
$$

Thus $\mathcal{L}$ is a weakly closed and convex subset of the (weakly) closed and convex cone of positive elements in $\mathbf{b a}(\mathbb{P})$.

Theorem 2.3 has its counterpart for coherent risk measures.

TheOREm $2.4[\mathrm{De}]$. Given a coherent risk measure $\varrho: L^{\infty}(\mathbb{P}) \rightarrow \mathbb{R}$, there is a convex $\sigma\left(\mathbf{b a}, L^{\infty}\right)$-closed set $P_{\mathrm{ba}} \subset$ ba of finitely additive probabilities such that

$$
\varrho(X)=\sup \left\{\langle-X, \mu\rangle \mid \mu \in P_{\mathrm{ba}}\right\},
$$

where

$$
\langle X, \mu\rangle=\mu(X)=\int_{\Omega} X d \mu \quad \text { for all } X \in L^{\infty}(\mathbb{P}) \text { and } \mu \in \mathbf{b a} .
$$

3. Conclusions. In our formulation of Theorem 2.3 we concentrated on positivity and convexity of the set $\mathcal{L}$ of positive linear extensions of the scalar base of the functional $F$ and skipped the fact that it is $\sigma\left(\mathbf{b a}, L^{\infty}\right)$-closed, but it clearly is, by Corollary 2.4 .

The considerable role played by the constant functions was demonstrated by Proposition 2.2. One can anticipate that many of the results obtained hold in linear lattices that have an analog of the space of constant functions. This problem and the problem of characterization of CRM functionals with arbitrary scalar bases will be dealt with in another publication.

\section{References}

[ADEH] P. Artzner, F. Delbaen, J.-M. Eber and D. Heath, Coherent measures of risk, Math. Finance 9 (1999), 203-228.

[Al] A. Alexiewicz, Functional Analysis, PWN, Warszawa, 1969 (in Polish).

[Bo] N. Bourbaki, Topological Vector Spaces, Chapters 1-5, Springer, Berlin, 1987.

[De] F. Delbaen, Coherent risk measures on general probability spaces, submitted. 
[Gr] A. Grothendieck, Topological Vector Spaces, Gordon and Breach, New York, 1973.

[Na] I. Namioka, Partially ordered linear topological spaces, Mem. Amer. Math. Soc. 24 (1957).

[YH] K. Yosida and E. Hewitt, Finitely additive measures, Trans. Amer. Math. Soc. 72 (1952), 46-66.

Faculty of Mathematics and Information Science

Warsaw University of Technology

Pl. Politechniki 1

00-661 Warszawa, Poland

E-mail: zbdudek@alpha.mini.pw.edu.pl

Received on 13.6.2001;

revised version on 15.10.2001 